\title{
Inhibition of cell viability by human IFN- $\beta$ is mediated by microRNA-431
}

\author{
TAKESHI TANAKA ${ }^{1}$, SHIGERU SUGAYA ${ }^{1}$, KAZUKO KITA $^{1}$, MAKOTO ARAI $^{2}$, TATSUO KANDA $^{2}$, \\ KATSUNORI FUJII ${ }^{3}$, FUMIO IMAZEKI ${ }^{2}$, KATSUO SUGITA ${ }^{4}$, OSAMU YOKOSUKA ${ }^{2}$ and NOBUO SUZUKI ${ }^{1}$ \\ Departments of ${ }^{1}$ Environmental Biochemistry, ${ }^{2}$ Medicine and Clinical Oncology, ${ }^{3}$ Pediatrics, \\ Graduate School of Medicine, Chiba University, 1-8-1 Inohana, Chiba 260-8670; \\ ${ }^{4}$ Faculty of Education, Chiba University, 1-33 Yayoichou, Chiba 263-8522, Japan
}

Received September 15, 2011; Accepted November 17, 2011

DOI: $10.3892 /$ ijo.2012.1345

\begin{abstract}
MicroRNAs (miRNAs) are small non-coding RNAs that inhibit gene expression by cleaving or hindering the translation of target mRNAs. We used microarray-based comparative transcriptome analysis to identify changes in miRNA expression and function between a human cell line, RSa, which is highly sensitive to HuIFN- $\beta$-mediated inhibition of cell viability, and its variant, the F-IF ${ }^{\mathrm{r}}$ cell line, which is relatively resistant to the cytokine. miR-431 expression was significantly higher in RSa cells compared with F-IFr cells. The addition of HuIFN- $\beta$ to $\mathrm{RSa}$ cultures reduced cell viability, down-regulated expression of IGFIR and IRS2 (putative miR-431 target genes), and inhibited the PI3K-Akt and MAPK pathways. The survival of F-IF cells was not reduced by HuIFN- $\beta$, but transient transfection with miR-431 precursors significantly decreased viability and concomitantly down-regulated IGFIR and IRS2 expression. In addition, the MAPK pathway, but not the PI3K-Akt pathway, was suppressed in F-IF ${ }^{\mathrm{r}}$ cells. Based on these results, we propose that, in RSa cells, HuIFN- $\beta$-induced miR-431 expression may down-regulate $I G F I R$ and $I R S 2$ expression, and consequently inhibit cell proliferation by suppressing the MAPK pathway.
\end{abstract}

\section{Introduction}

MicroRNAs (miRNAs) are small non-coding RNAs that regulate gene expression by base pairing specifically with nucleotides in the 3 ' untranslated regions of target mRNAs or by directing mRNA degradation (1). MiRNAs play important roles in cell proliferation, apoptosis, and differentiation $(2,3)$. In particular, miRNAs that regulate cell proliferation have been implicated in various cancers. Numerous studies have revealed differences in the expression of various miRNAs between tumors and normal

Correspondence to: Dr Takeshi Tanaka or Dr Nobuo Suzuki, Department of Environmental Biochemistry, Graduate School of Medicine, Chiba University, 1-8-1 Inohana, Chiba 260-8670, Japan E-mail: take64@faculty.chiba-u.jp

E-mail: nobuo@faculty.chiba-u.jp

Key words: microRNA, human IFN- $\beta$, IGF signaling pathway, cell viability, cancer therapy tissues (4), suggesting that miRNAs can function as either tumor suppressors or oncogenes in human cancers. In addition, there are a number of reports demonstrating that certain miRNAs participate in the activity of various anti-cancer drugs. For example, the combination of miR-145 and 5-fluorouracil (5-FU) has an anti-tumor effect on breast cancer (5). In a non-small cell lung cancer cell line (A549), cisplatin sensitivity was increased by up-regulating miR-451 expression (6). In contrast, miR-21 induced resistance to the anti-cancer effects of IFN- $\alpha / 5-F U$ combination therapy in hepatocellular carcinoma cells (7). Human interferons (HuIFNs) are a family of cytokines that have various biological functions, including anti-viral and antitumor activity (8). They also regulate basic cellular functions including growth, differentiation, and immune reactivity (9-11). HuIFNs induce a cascade of events leading to an increase in the expression of various genes, including those responsible for the biological effects of IFNs (9-13). HuIFNs are divided into type I, II, and III subtypes, based in part on the differential use of unique receptors through which they mediate their biological effects. HuIFN- $\alpha$ and $-\beta$ are type I IFNs. HuIFN- $\beta$ has proven useful as an anti-neoplastic drug, although the role of miRNAs in this function is not clear. In this study, we focused on the miRNAs that were induced by HuIFN- $\beta$ using the HuIFN- $\beta$ sensitive cell line, RSa, and its resistant variant, the $\mathrm{F}_{-} \mathrm{IF}^{\mathrm{r}}$ cell line. Our aim was to identify miRNAs that are up-regulated by HuIFN- $\beta$ and are responsible for suppressing cell proliferation in human cells.

\section{Materials and methods}

Cell lines and culture conditions. RSa cells were established from human embryo-derived fibroblasts by double infection with Simian virus 40 and Rous sarcoma virus. These cells are highly sensitive to the ability of HuIFN- $\beta$ to inhibit cell viability. The $\mathrm{F}-\mathrm{IF}^{\mathrm{r}}$ line is a variant with increased resistance to HuIFN- $\beta$. Both cell lines were cultured in Eagle's minimal essential medium (EMEM; Nissui, Tokyo, Japan) containing $10 \%$ calf serum (CS; Invitrogen, Carlsbad, CA, USA) at $37^{\circ} \mathrm{C}$ in a humidified atmosphere containing $5 \% \mathrm{CO}_{2}$.

RNA extraction. Total RNA was extracted using TRIzol reagent (Invitrogen) according to the manufacturer's instructions. 
Microarray analysis. We analyzed miRNA expression using Genopal ${ }^{\circledR}$-MICH chips (Mitsubishi Rayon, Yokohama, Japan), equipped with 188 oligonucleotide DNA probes in hollow plastic fibers for detection of human miRNAs. Hybridization signals were analyzed using a DNA chip analyzer according to the manufacturer's instructions.

Estimation of miRNA expression levels. Quantitative RT-PCR for three miRNAs (miR-193b, miR-381, and miR-431) was carried out using the TaqMan MicroRNA Assay (Applied Biosystems, Foster City, CA, USA). Total RNA (30 ng) from $\mathrm{RSa}$ and $\mathrm{F}-\mathrm{IF}^{\mathrm{r}}$ cells were reverse-transcribed using the TaqMan Reverse Transcription kit (Applied Biosystems) according to the manufacturer's protocol. For normalization, each miRNA was amplified on the same plate with the reference miRNA, RNU6B, and we calculated changes in expression levels relative to this standard.

HuIFN- $\beta$ and PI3K inhibitor LY294002 treatment. Recombinant human IFN- $\beta$ was obtained from PeproTech (Rocky Hill, NJ, USA). PI3K inhibitor LY294002 was obtained from Cosmo Bio (Tokyo, Japan). Briefly, logarithmically growing RSa and $\mathrm{F}-\mathrm{IF}^{\mathrm{r}}$ cells were plated at a density of $5 \times 10^{5}$ cells $/ 35-\mathrm{mm}$ culture dish, and after $24 \mathrm{~h}$, the medium was replaced with fresh EMEM or the same medium containing HuIFN- $\beta$ (at either 100 or $500 \mathrm{IU} / \mathrm{ml})$ or PI3K inhibitor LY294002 (75 $\mu \mathrm{M})$.

Measurement of cell viability. Cell viability was measured from the average of 6 MTS assays per sample, quantified by absorbance at $490 \mathrm{~nm}$ in a microplate colorimeter using the CellTiter 96 AQueous One Solution Reagent (Promega, Madison, WI, USA) according to the manufacturer's instructions, for each time point.

Transfection of miRNA molecules. MiRNA precursors that mimic miR-381 or miR-431, a control non-specific miRNA (Pre-miR Negative Control), and anti-miRNA molecules were obtained from Ambion (Austin, TX, USA). Using siPORT NeoFX (Ambion), miRNA precursors were transfected into F-IF ${ }^{r}$ cells and anti-miRNA molecules into RSa cells, according to the manufacturer's protocol. For siRNA transfection, cells (plated to reach $80 \%$ confluence after 12 or $24 \mathrm{~h}$ ) were treated with siRNAs complementary to each target gene or a control using siPORT NeoFX. Predicted target genes for miRNA were identified using TargetScan (http://www.targetscan.org).

Western blotting. Cells grown to $80 \%$ confluence were treated with HuIFN- $\beta$, pre-miR-431 or anti-miR-431. After treatment, cells were washed three times with ice-cold PBS. Equal amounts of protein were separated by SDS-PAGE and transferred to polyvinylidene fluoride membranes (ATTO, Tokyo, Japan). Membranes were blocked in 5\% non-fat dried milk in PBST and incubated with primary antibody. The following antibodies were used: IGF-1R $\alpha$, IGF-1R $\beta$, IRS2, and total Akt1/2/3 from Santa Cruz Biotechnology, Santa Cruz, CA, USA; p-Akt (phospho-Akt, $\mathrm{Thr}^{308}$ ), p-Akt (phospho-Akt, Ser ${ }^{473}$ ), p44/42 MAPK (Erk1/2), and phosphor-p44/42 MAPK (Erk1/2), from Cell Signaling Technology (Danvers, MA, USA). GAPDH (Sigma, St. Louis, MO, USA) was used as an internal control. Secondary antibodies were conjugated to horseradish peroxidase and immunoreactive proteins were detected using the ECL-plus system (Amersham, Piscataway, NJ, USA).

Estimation of miRNA target gene expression. Total RNA (30 ng) from F-IF ${ }^{\mathrm{r}}$ cells was reverse-transcribed using Super Script III First-Strand Synthesis SuperMix (Invitrogen) according to the manufacturer's protocol. Steady-state expression levels of IGFIR and IRS 2 mRNAs were evaluated by quantitative RT-PCR using Fast SYBR Green Master Mix and the following oligonucleotide primers and annealing temperatures: $I G F 1 R$, 5'-CATCGACATCCGCAACGA-3' (forward) and 5'-CCCTCGATCACCGTGCA-3' (reverse) at 50 ${ }^{\circ}$; IRS2, 5'-GT ACCTGATCGCCCTCTAC-3' (forward) and 5'-AGGCACA GACGGTACAC-3' (reverse) at $50^{\circ} \mathrm{C}$. Expression of each gene was normalized to a GAPDH control: 5'-ACCCACTCCTCCA CCTTTG-3' (forward) and 5'-CTCTTGTGCTCTTGCTGG G-3' (reverse).

\section{Results}

Comparison of miRNA expression levels between RSa and F-IF cells. MiRNA expression levels in RSa cells were compared to those in F-IF ${ }^{\mathrm{r}}$ cells by microarray analysis. The levels of miR-193b, miR-381, and miR-431 were significantly higher in RSa cells, being over 5-fold greater than in F-IFr cells (data not shown). Quantitative RT-PCR analysis of the three miRNAs revealed a significantly higher expression of miR-381 and miR-431 (3.56- and 3.81-fold greater, respectively) in RSa cells relative to $\mathrm{F}-\mathrm{IF}^{\mathrm{r}}$ cells.

Effect of HuIFN- $\beta$ on cell viability and miR-431 expression. When HuIFN- $\beta$ was added to culture medium at a concentration $500 \mathrm{IU} / \mathrm{ml}$, a significant suppression of RSa cell viability was observed $24 \mathrm{~h}$ later (Fig. 1A). In contrast, F-IF ${ }^{\mathrm{r}}$ cell viability was not significantly suppressed by HuIFN- $\beta$, at any dose up to $500 \mathrm{IU} / \mathrm{ml}$, nor by any duration up to $24 \mathrm{~h}$ (Fig. 1B). HuIFN- $\beta$ (500 IU/ml) induced a significant increase in miR-431 expression in RSa cells relative to F-IF ${ }^{\mathrm{r}}$ cells $24 \mathrm{~h}$ following addition, but it did not affect miR-381 expression (Fig. 1C).

Examination of the role of miR-431 on cell viability. In order to examine whether increased expression of miR-431 results in reduced cell viability, $\mathrm{F}_{-} \mathrm{IF}^{\mathrm{r}}$ cells were transiently transfected with miR-431, this resulted in a significant suppression of cell viability $24 \mathrm{~h}$ later (Fig. 2A). No significant effect on viability was observed following transfection with miR-381 (Fig. 2A). When RSa cells were treated with both HuIFN- $\beta$ and anti-miR-431, cell proliferation was not suppressed at $24 \mathrm{~h}$ (Fig. 2B).

Search for miR-431 target genes. Predicted target genes for miR-431 were identified with TargetScan (Table I). Among these genes, we focused on the cell viability-related genes, $I G F 1 R$ (insulin-like growth factor 1 receptor) and IRS2 (insulin receptor substrate 2). Schematic representation of $I G F 1 R$ and IRS 2 mRNAs, showing the predicted miR-431 binding sites located in their 3' UTR, are given in Fig. 3A. To determine the effects of reducing IGFIR or IRS $2 \mathrm{mRNA}$ levels on cell viability, we assessed the viability of $F-I^{r}{ }^{r}$ cells treated with gene-specific siRNAs or a non-targeting control siRNA. Treatment with the 

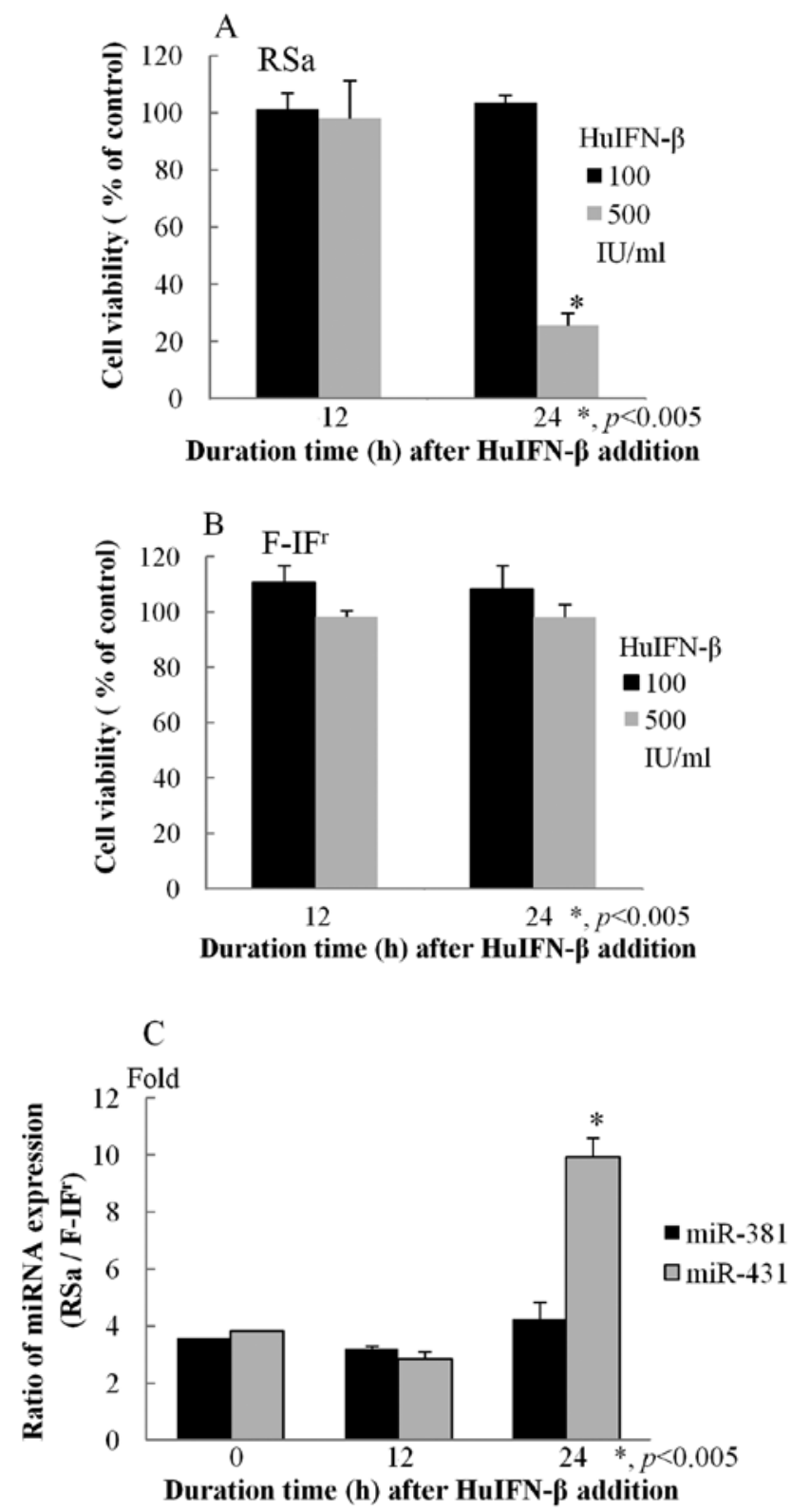

Figure 1. Effect of HuIFN- $\beta$ treatment on cell viability and expression levels of miRNAs. (A and B), HuIFN- $\beta$ was added to the culture medium of RSa and F-IFr cells, at $100 \mathrm{IU} / \mathrm{ml}$ (black) or $500 \mathrm{IU} / \mathrm{ml}$ (grey) ("p $<0.005$, t-test). (C), Quantitative RT-PCR was performed to examine the effects of a 24-h HuIFN- $\beta$ ( $500 \mathrm{IU} / \mathrm{ml}$ ) treatment on miR-381 (black) and miR-431 (grey) expression in RSa cells relative to F-IFr cells. MiRNA expression is presented as a fold difference with respect to F-IFr cells, with the value for F-IFr cells set at a fold change of 1 . Results are presented as the means $\pm \mathrm{SD}^{*} \mathrm{p}<0.005$.
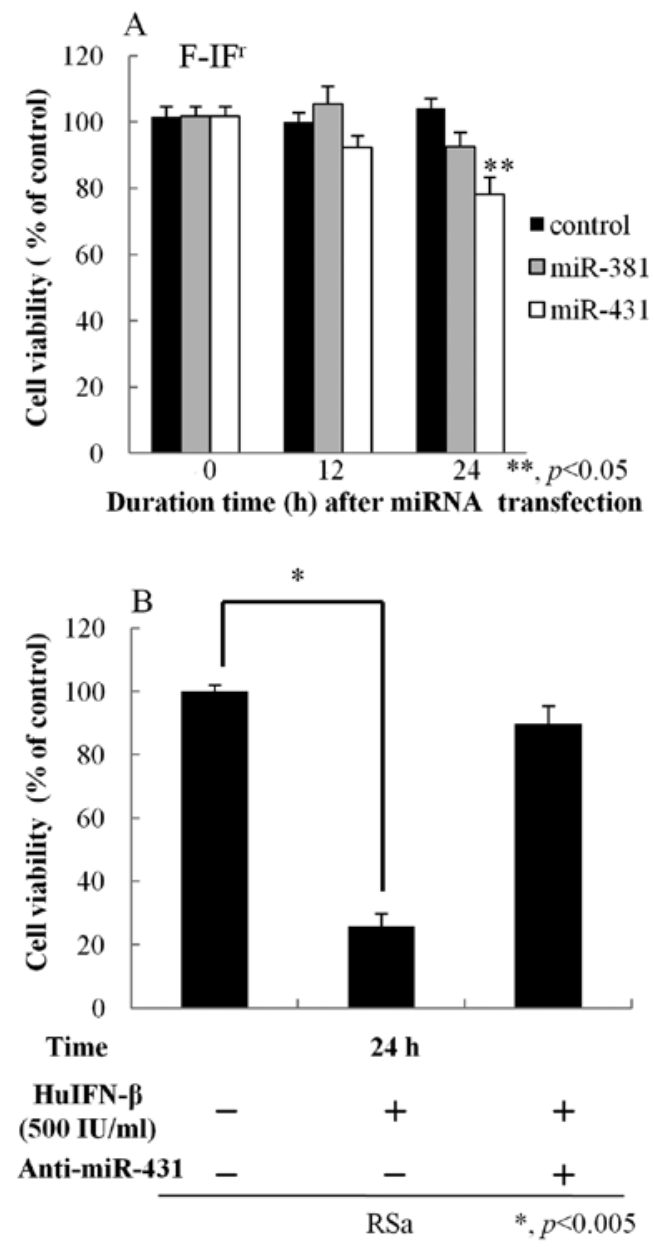

Figure 2. Effects of miRNA transient transfection or HuIFN- $\beta$ treatment with or without anti-miR-431 addition on cell viability. (A), Transient transfection with miR-381 (grey) or miR-431 (white) in F-IFr cells ( ${ }^{* *} \mathrm{p}<0.05$, t-test). (B), Changes in cell viability in RSa cells treated with HuIFN- $\beta$ with or without anti-miR-431. Results are presented as the means $\pm \mathrm{SD}$. ${ }^{*} \mathrm{p}<0.005,{ }^{* *} \mathrm{p}<0.05$.

siRNAs led to a significant suppression of cell viability relative to control cells (Fig. 3B).

Examination of IGFIR signaling pathways. When RSa cells were treated with HuIFN- $\beta$ (500 IU/ml), a reduction in protein levels of IGF1R $\alpha / \beta$ and IRS 2 was consistently observed after $24 \mathrm{~h}$ (Fig. 4A). A similar reduction was also detected in F-IF cells transfected with miR-431 precursors (Fig. 4B). However,

Table I. Predicted miR-431 targets involved in cell viability. ${ }^{\mathrm{a}}$

\begin{tabular}{|c|c|c|c|c|c|c|c|c|c|c|}
\hline \multirow[b]{2}{*}{$\begin{array}{l}\text { Gene } \\
\text { symbol }\end{array}$} & \multirow[b]{2}{*}{ Gene name } & \multicolumn{4}{|c|}{ Conserved sites } & \multicolumn{4}{|c|}{ Poorly conserved sites } & \multirow[b]{2}{*}{$\begin{array}{c}\text { Total cont. } \\
\text { score }\end{array}$} \\
\hline & & Total & 8 -mer & 7-mer-m8 & 7-mer-1A & Total & 8 -mer 7 & 7-mer-m8 & 7 -mer-1A & \\
\hline IGF1R & Insulin-like growth factor 1 receptor & 1 & 1 & 0 & 0 & 0 & 0 & 0 & 0 & -0.17 \\
\hline IRS2 & Insulin receptor substrate 2 & 1 & 1 & 0 & 0 & 0 & 0 & 0 & 0 & -0.37 \\
\hline
\end{tabular}

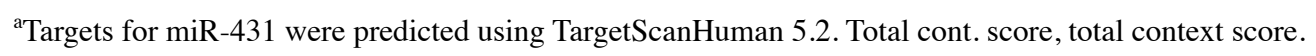


A

\begin{tabular}{|c|c|c|}
\hline $\begin{array}{l}\text { Position 1613-1619 of } \\
\text { IGFIR 3' UTR }\end{array}$ & 5' GUUACAGU & GCAAGAC \\
\hline has-miR-431 & $3^{\circ}$ ACUGCCGG & CGUUCUG \\
\hline $\begin{array}{l}\text { Position 1631-1637 of } \\
\text { IRS2 } 3^{\prime} \text { UTR }\end{array}$ & 5' AUUGUAUU & GCAAGAC \\
\hline has-miR-431 & G & CGUUCUS \\
\hline
\end{tabular}

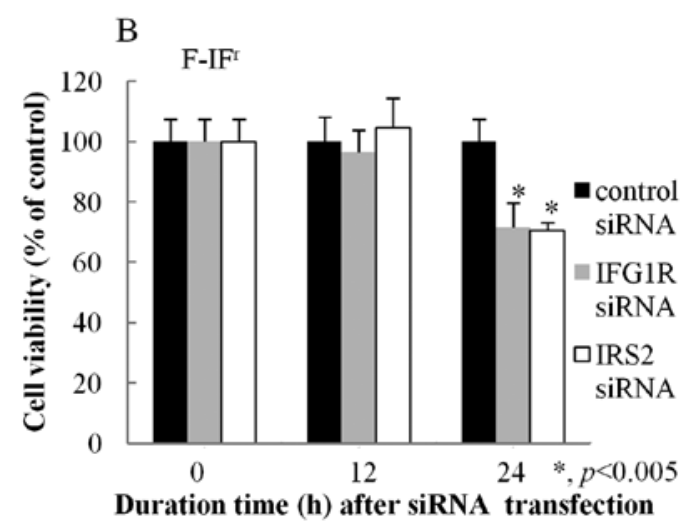

Figure 3. Cell viability after transient transfection with siRNA (IGF1R or IRS2) in F-IF ${ }^{\mathrm{r}}$ cells. (A), Sites of miR-431 seed matching in the IGF1R and IRS2 3' UTRs. (B), Transfection with control siRNA (black) or IGFIR (grey) or IRS2 (white) siRNA in F-IFr cells, respectively. A significant suppression of cell viability was observed in F-IF ${ }^{\mathrm{r}}$ cells at $24 \mathrm{~h}$ relative to the control. Results are presented as means $\pm \mathrm{SD}$. ${ }^{*} \mathrm{p}<0.005$.

A

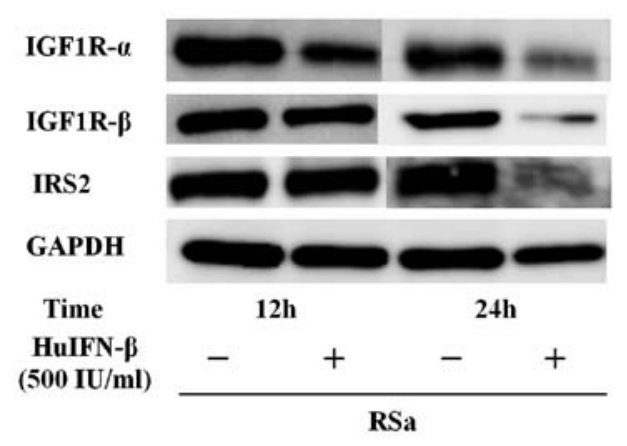

C

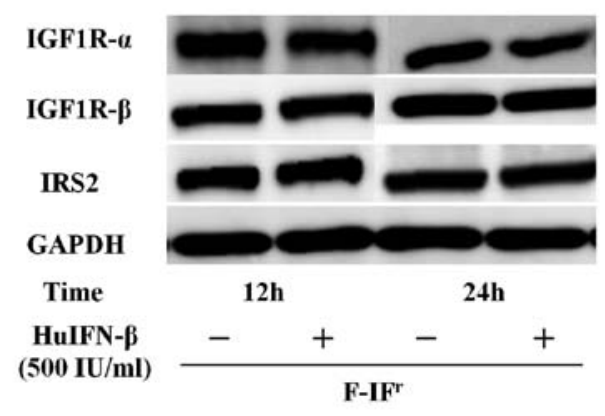

B

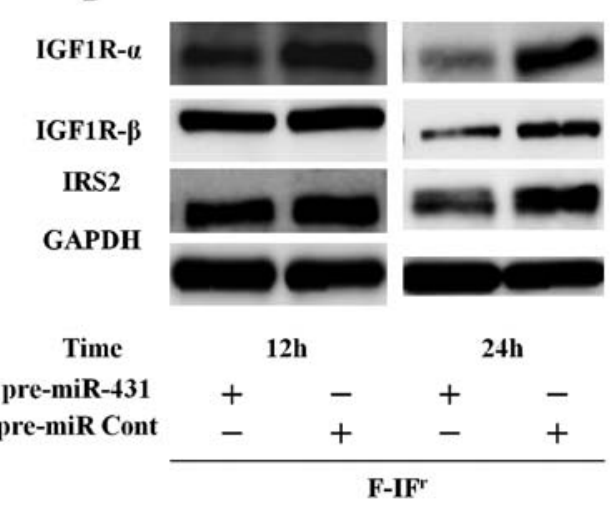

$\mathrm{D}$

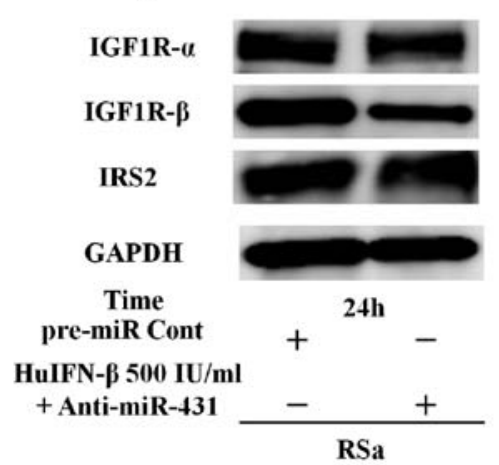

Figure 4. Effects of HuIFN- $\beta$ and miR-431 on IGF1R and IRS2 expression. (A and C), Immunoblots of IGF1R ( $\alpha / \beta$ ) and IRS2 proteins in RSa and F-IF ${ }^{\mathrm{r}}$ cells with or without HuIFN- $\beta(500 \mathrm{IU} / \mathrm{ml})$ treatment. (B), The levels of IGF1R $(\alpha / \beta)$ and IRS2 in F-IF ${ }^{\mathrm{r}}$ cells transfected with pre-miR-431 and pre-miR negative control precursors for 12 or 24 h. (D), Expression levels of IGF1R and IRS2 in RSa cells treated with HuIFN- $\beta$ (500 IU/ml) and anti-miR-431 for 24 h. GAPDH was used as a loading control.

no apparent change in protein levels was observed when F-IF cells were treated with HuIFN- $\beta$ (Fig. 4C). HuIFN- $\beta$ (500 IU/ $\mathrm{ml}$ ) and anti-miR-431 treatment resulted in a significant increase in the expression of IGF1R and IRS2 in RSa cells (Fig. 4D).

Since IGF1R is a major player in the PI3K-Akt and MAPK signaling pathways, we investigated the effects of miR-431mediated IGFIR down-regulation on PI3K-Akt and MAPK phosphorylation. In RSa cells, protein levels of total Akt, p-Akt $\mathrm{Thr}^{308}$, p-Akt Ser ${ }^{473}$, total Erk1/2, and p-Erk1/2 were reduced after $24 \mathrm{~h}$ of HuIFN- $\beta$ (500 IU/ml) treatment (Fig. 5A). In contrast, the levels of these proteins were not changed in F-IFr cells (Fig. 5B). When F-IFr cells were transfected with miR-431 precursors, reductions in the levels of total Erk1/2 and $\mathrm{p}$-Erk1/2 were observed after $24 \mathrm{~h}$ of transfection, while the levels of total Akt, p-Akt $\mathrm{Thr}^{308}$, and p-Akt Ser ${ }^{473}$ were 
A

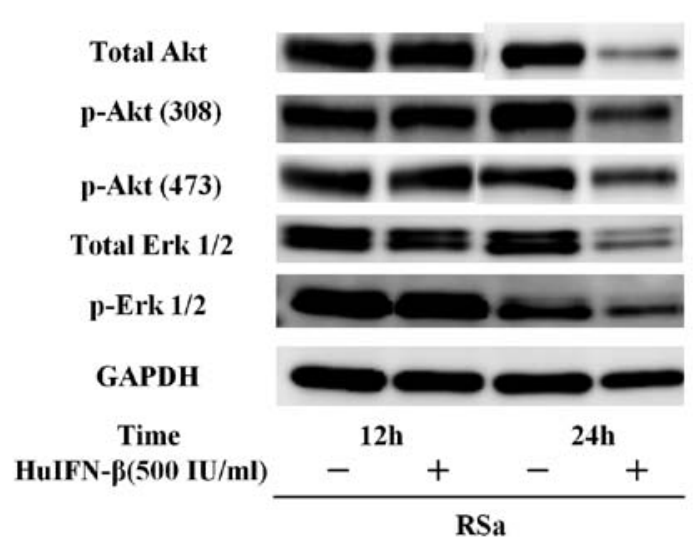

C

Total Akt
p-Akt (308)

p-Akt (473)

Total Erk1/2

p-Erk 1/2

GAPDH

\begin{tabular}{c}
\multicolumn{1}{c}{ Time } \\
pre-miR-431 \\
pre-miR Cont
\end{tabular}

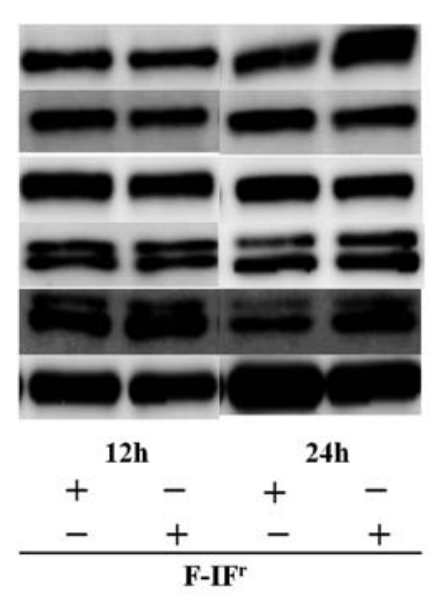

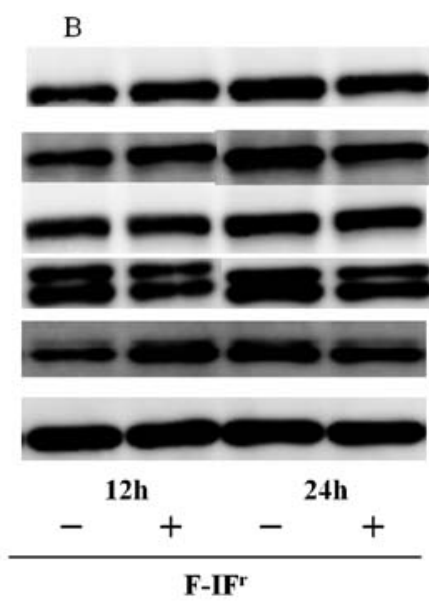

D

Total Akt

p-Akt (308)

p-Akt (473)

Total Erk1/2

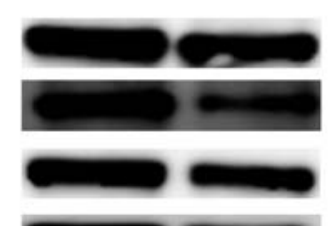

p-Erk 1/2

GAPDH

Time

pre-miR Cont

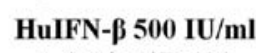

+Anti-miR-431

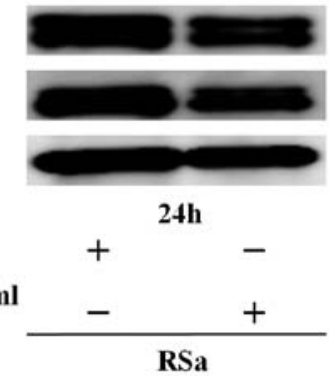

Figure 5. Inhibition of the PI3K-Akt and MAPK pathways targeted by HuIFN- $\beta$ and miR-431. (A and B), Immunoblots of total Akt, p-Akt (Ser ${ }^{473}$, Thr ${ }^{308}$ ), total Erk1/2, and p-Erk1/2 in RSa and F-IFr cells with or without treatment with HuIFN- $\beta(500 \mathrm{IU} / \mathrm{ml})$ for 12 or $24 \mathrm{~h}$. (C), The levels of total Akt, p-Akt (Ser $\left.{ }^{473}, \mathrm{Thr}^{308}\right)$, total Erk1/2, and p-Erk1/2 in F-IFr cells which were transfected with pre-miR-431 or pre-miR control precursors for 12 or $24 \mathrm{~h}$. (D), The levels of total Akt, p-Akt $\left(\mathrm{Ser}^{473}, \mathrm{Thr}^{308}\right)$, total Erk1/2, and p-Erk1/2 in RSa cells which were treated with HuIFN- $\beta$ (500 IU/ml) and anti-miR-431 for $24 \mathrm{~h}$. GAPDH was used as a loading control.

not significantly altered (Fig. 5C). Knockdown of miR-431 by anti-miR-431 and simultaneous treatment with HuIFN- $\beta$ $(500 \mathrm{IU} / \mathrm{ml})$ removed the suppression of total Akt, p-Akt $\mathrm{Thr}^{308}$, p-Akt Ser ${ }^{473}$, total Erk1/2, and p-Erk1/2 expression in RSa cells (Fig. 5D).

Suppression of miR-431 target genes. Transient transfection with miR-431 precursors significantly suppressed IGF1R and IRS2 expression in $\mathrm{F}-\mathrm{IF}^{\mathrm{r}}$ cells after $24 \mathrm{~h}$ relative to control-transfected cells (Fig. 6A). Treating RSa cells with HuIFN- $\beta$ (500 IU/ml) also significantly diminished $I G F I R$ and $I R S 2$ expression after 24 h relative to control cells (Fig. 6B). However, when RSa cells were treated with both HuIFN- $\beta$ (500 IU/ml) and anti-miR-431, $I G F 1 R$ and $I R S 2$ expression were not significantly affected (Fig. 6B).

Effect of PI3K inhibitor on cell viability. F-IF $\mathrm{F}^{\mathrm{r}}$ cells were transfected with miR-431 precursors and/or the PI3K inhibitor LY294002. Proliferation was reduced by approximately $20 \%$ when cells were transfected with miR-431 precursors. However, it was suppressed approximately $30 \%$ when cells were transfected with miR-431 precursors and treated with LY294002 at the same time (Fig. 7).

\section{Discussion}

There are numerous reports of the involvement of miRNAs in regulating cell proliferation in a multitude of cancer cell lines (4). We focused on a few miRNAs whose expression levels were altered by the addition of HuIFN- $\beta$ in a non-cancer HuIFN- $\beta$ sensitive cell line, RSa, and its variant HuIFN- $\beta$ resistant cell line, F-IF .

Based on microarray analysis, we focused on three miRNAs (miR-193b, miR-381, and miR-431) whose expression levels were up-regulated more than 5-fold in RSa cells relative to F-IF ${ }^{r}$ cells. Among these, miR-381 and miR-431 were also up-regulated according to quantitative RT-PCR. In addition, HuIFN- $\beta$ treatment increased miR-431 expression and reduced cell viability in RSa cells (Fig. 1A and C). In contrast, transfection of miR-431 precursors into F-IF ${ }^{\mathrm{r}}$ cells significantly reduced cell viability (Fig. 2A). These results suggest that miR-431 plays a role in regulating cell viability.

We then sought to determine whether miRNA-regulated signaling pathways modulated cell viability. Previous reports revealed that some signaling pathways that affect cell viability are regulated by miRNAs (14-19). We decided to focus on one of these - the IGF signaling pathway. The factors that activate IGF 

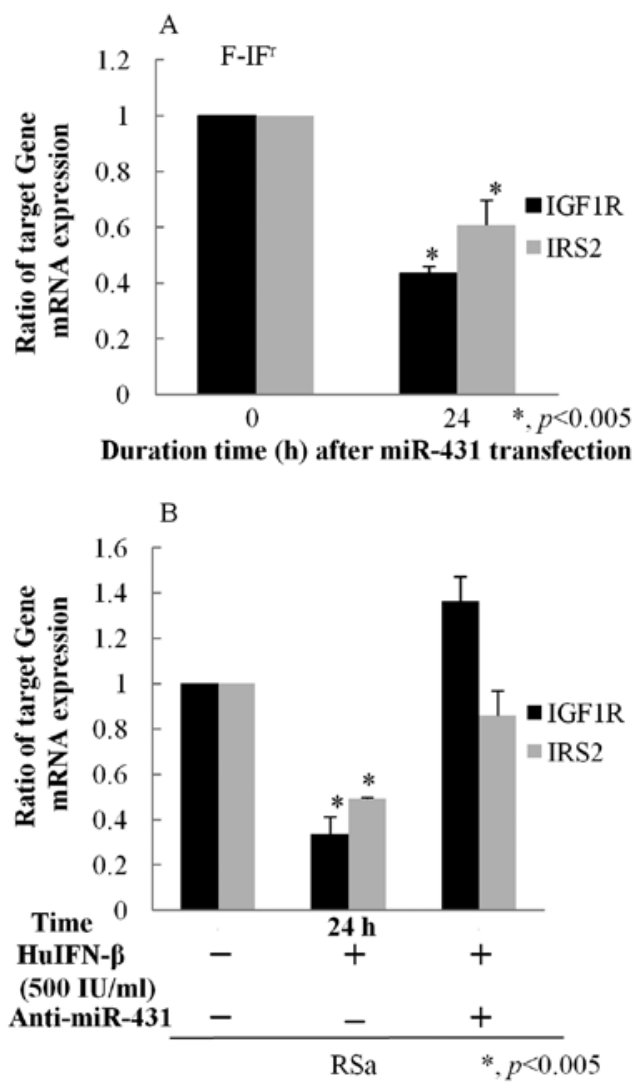

Figure 6. IGFIR and IRS2 mRNA expression. (A), Quantitative RT-PCR was performed to examine the effects of miR-431 on IGF1R (black) and IRS2 (grey) mRNA levels in F-IFr cells that were transfected with pre-miR-431 precursors or the pre-miR control. mRNA expression levels are presented as a fold difference with respect to F-IFr cells transfected with the pre-miR control, which was set at a fold change of 1. (B), IGF1R (black) and IRS2 (grey) mRNA levels were analyzed by quantitative RT-PCR after $24 \mathrm{~h}$ following HuIFN- $\beta$ addition in combination with anti-miR-431 treatment. mRNA expression levels are presented as a fold difference with respect to control RSa cells [HuIFN- $\beta(-)$, anti-miR-431(-)] which was set at a fold change of 1 . Results are presented as the means $\pm \mathrm{SD}$. ${ }^{*} \mathrm{p}<0.005$

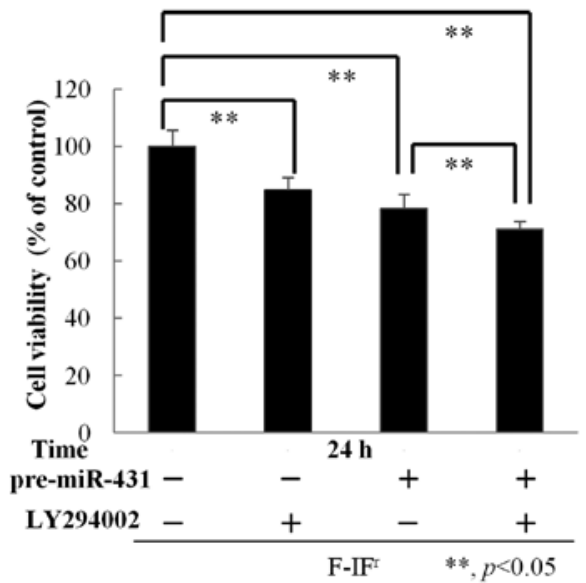

Figure 7. Effects of pre-miR-431 and the PI3K inhibitor on cell viability F-IF ${ }^{r}$ cells were transfected with pre-miR-431 precursors and/or treated with the PI3K inhibitor LY294002. Results are presented as means \pm SD. ${ }^{* *} \mathrm{p}<0.05$

signaling pathways and promote cell proliferation in cancers have been investigated by others (20). For numerous cancers, the activation of IGF signaling pathways contributes to cell proliferation. Therefore, the suppression of this pathway should inhibit cancer cell proliferation. In tongue squamous cell carcinoma, miR-7 levels are reduced, but the ectopic expression of miR-7 significantly down-regulates IGF1R expression, at both the mRNA and protein levels (21). Thus, a miRNA can suppress a cell growth-signaling pathway.

In the present study, we examined the miR-431 target genes that are suspected of suppressing cell viability, and focused on IGFIR and IRS2, which are functional targets of miR-431. IGF1R is a receptor tyrosine kinase that consists of heterotetramers $\left(\alpha_{2} \beta_{2}\right)$ held together by disulfide bonds, and it mediates IGF1-induced signaling. IRS 2 is the main effector of the proliferative signals triggered by IGF receptors (22). According to Western blot analysis, IGF1R and IRS2 are down-regulated in RSa cells by HuIFN- $\beta$ and in F-IF ${ }^{\mathrm{r}}$ cells by transfection with miR-431 precursors (Fig. 4A and B). These observations suggest that increased levels of miR-431 inhibit IGF1R and $I R S 2$ expression in RSa cells. In addition, we hypothesized that miR-431-mediated IGFIR and IRS2 down-regulation would be accompanied by the inhibition of the PI3K-Akt and MAPK pathways. Indeed, HuIFN- $\beta$ suppressed both signaling pathways in RSa cells (Fig. 5A). Transfection with miR-431 precursors inhibited the MAPK pathway in $\mathrm{F}-\mathrm{IF}^{\mathrm{r}}$ cells, although the PI3K-Akt pathway was not affected (Fig. 5C).

There are a number of clinical reports on the therapeutic use of HuIFN- $\beta$. For example, combination therapy with HuIFN- $\beta$ and Ranimustive has been particularly useful for the treatment of malignant gliomas in Japan (23). In cases of newly diagnosed primary glioblastoma multiforme, HuIFN- $\beta$ and temozolomide combination therapy is significantly associated with a favorable outcome (24). However, there are many hurdles facing the use of HuIFN- $\beta$ as an anti-cancer agent, particularly for HuIFN- $\beta$ resistant cancers. For example, NC65 tumors (a human renal cell carcinoma) transfected with recombinant HuIFN- $\beta$ did not shrink and failed to undergo apoptosis (25). Thus, in clinical cases in which enhanced HuIFN- $\beta$ sensitivity is desirable or in which resistance to HuIFN- $\beta$ is encountered, the delivery of miR-431 precursors to the cancerous tissue, in combination with a PI3K inhibitor, may effectively suppress cancer growth.

In conclusion, our results demonstrate that miR-431, which is up-regulated by HuIFN- $\beta$ in RSa cells, suppresses the IGF1R signaling pathway. Delivery of miR-431 and a PI3K inhibitor in a combination therapeutic approach may represent an effective strategy for the treatment of HuIFN- $\beta$ resistant cancers.

\section{Acknowledgments}

The authors thank Mrs. Satomi Hasegawa for her excellent technical assistance. This research project was supported by the Ministry of Health, Labour and Welfare (Science Research Grants for intractable diseases in Japan; H22-intractable diseases-120), the Smoking Research Foundation, the Tsuchiya Foundation, the Kieikai Research Foundation, the Ministry of Health, Labour and Welfare for the Intractable Diseases Treatment Research Program, and the Japan Society for the Promotion of Science (Japan).

\section{References}

1. He L and Hannon GJ: MicroRNAs: small RNAs with a big role in gene regulation. Nat Rev Genet 5: 522-531, 2004. 
2. Ambros V: The functions of animal microRNAs. Nature 431: 350-355, 2004

3. Bartel DP: MicroRNAs: genomics, biogenesis, mechanism, and function. Cell 116: 281-297, 2004.

4. Lu J, Getz G, Miska EA, et al: MicroRNA expression profiles classify human cancers. Nature 2435: 834-838, 2005.

5. Kim SJ, Oh JS, Shin JY, et al: Development of microRNA-145 for therapeutic application in breast cancer. J Control Release (In press).

6. Bian HB, Pan X, Yang JS, Wang ZX and De W: Upregulation of microRNA-451 increases cisplatin sensitivity of non-small cell lung cancer cell line (A549). J Exp Clin Cancer Res 30: 20, 2011

7. Tomimaru Y, Eguchi H, Nagano H, et al: MicroRNA-21 induces resistance to the anti-cancer effect of interferon- $\alpha / 5$-fluorouracil in hepatocellular carcinoma cells. Br J Cancer 103: 1617-1626, 2010.

8. Pfeffer LM: Mechanisms of interferon action. CRC Press, Boca Raton, FL, 1987.

9. Petska S, Langer AJ, Zoon K and Samuel C: Interferons and their action. Annu Rev Biochem 56: 727-777, 1987.

10. Taniguchi $\mathrm{T}$ and Takaoka A: The interferon-alpha/beta system in antiviral responses: a multimodal machinery of gene regulation by the IRF family of transcription factors. Curr Opin Immuno 14: 111-116, 2002

11. Biron CA: Interferon alpha and beta as immune regulators - a new look. Immunity 14: 661-664, 2001.

12. Revel $\mathrm{M}$ and Chebath J: Interferon-activated genes. Trends Biochem Sci 11: 166-170, 1986.

13. Williams BR: Transcriptional regulation of interferon-stimulated genes. Eur J Biochem 200: 1-11, 1991.

14. Kogo R, Mimori K, Tanaka F, Komune S and Mori M: Clinical significance of miR-146a in gastric cancer cases. Clin Cancer Res 17: 4277-4284, 2011.

15. Saydam O, Senol O, Wurdinger T, et al: miRNA-7 atttenuation in Schwannoma tumors stimulates growth by upregulating three oncogenic signaling pathways. Cancer Res 71: 852-861, 2011.

16. Chou YT, Lin HH, Lien YU, et al: EGFR promotes lung tumorigenesis by activating miR-7 through a Ras/ERK/Myc pathway that targets the Ets2 transcriptional repressor ERF. Cancer Res 70: 8822-8831, 2010.
17. Giles KM, Barker A, Zhang PM, Epis MR and Leedman PJ: MicroRNA regulation of growth factor receptor signaling in human cancer cells. Methods Mol Biol 676: 147-163, 2011.

18. Guo C, Sah JF, Beard L, Willson JK, Markowitz SD and Guda K: The non-coding RNA, miR-126, suppresses the growth of neoplastic cells by targeting phosphatidylinositol 3-kinase signaling and is frequently lost in colon cancers. Gene Chromosomes Cancer 47: 939-946, 2008

19. Kefas B, Godlewski J, Comeau L, et al: microRNA-7 inhibits the epidermal growth factor receptor and the Akt pathway and is down-regulated in glioblastoma. Cancer Res 68: 3566-3572, 2008.

20. La Rocca G, Badin M, Shi B, et al: Mechanism of growth inhibition by microRNA 145: the role of the IGF-I receptor signaling pathway. J Cell Physiol 220: 485-491, 2009.

21. Jiang L, Liu X, Chen Z, et al: MicroRNA-7 targets IGF1R (insulinlike growth factor 1 receptor) in tongue squamous cell carcinoma cells. Biochem J 432: 199-205, 2010.

22. Kwon J, Stephan S, Mukhopadhyay A, et al: Insulin receptor substrate-2 mediated insulin-like growth factor-I receptor overexpression in pancreatic adenocarcinoma through protein kinase Co. Cancer Res 69: 1350-1357, 2009.

23. Wakabayashi T, Hatano N, Kajita Y, et al: Initial and maintenance combination treatment with interferon-beta, MCNU (Ranimustine), and radiotherapy for patients with previously untreated malignant glioma. J Neurooncol 49: 57-62, 2000.

24. Motomura K, Natsume A, Kishida Y, et al: Benefits of interferon- $\beta$ and temozolomide combination therapy for newly diagnosed primary glioblastoma with the unmethylated MGMT promoter. Cancer 117: 1721-1730, 2011.

25. Yamamoto K, Mizutani Y, Nakanishi H, et al: Significant antitumor activity of cationic multilamellar liposomes containing human interferon- $\beta$ gene in combination with 5-fluorouracil against human renal cell carcinoma. Int J Oncol 33: 565-571, 2008 . 